Algebraic 83 Geometric $\mathcal{T}$ opology

Volume 1 (2001) 39-55

Published: 9 December 2000

\title{
ATG
}

\section{An expansion of the Jones representation of genus 2 and the Torelli group}

\author{
YASUSHI KASAHARA
}

\begin{abstract}
We study the algebraic property of the representation of the mapping class group of a closed oriented surface of genus 2 constructed by V F R Jones [9]. It arises from the Iwahori-Hecke algebra representations of Artin's braid group of 6 strings, and is defined over integral Laurent polynomials $\mathbb{Z}\left[t, t^{-1}\right]$. We substitute the parameter $t$ with $-e^{h}$, and then expand the powers $e^{h}$ in their Taylor series. This expansion naturally induces a filtration on the Torelli group which is coarser than its lower central series. We present some results on the structure of the associated graded quotients, which include that the second Johnson homomorphism factors through the representation. As an application, we also discuss the relation with the Casson invariant of homology 3-spheres.
\end{abstract}

AMS Classification 57N05; 20F38, 20C08, 20F40

Keywords Jones representation, mapping class group, Torelli group, Johnson homomorphism

\section{Introduction}

Let $\Sigma_{2}$ be a closed oriented surface of genus 2 , and let $\mathcal{M}_{2}$ be its mapping class group. In [9], V F R Jones constructed a finite dimensional linear representation of $\mathcal{M}_{2}$. We call this the Jones representation of genus 2 , and denote it by $\rho$ (see section 2.3 for the precise definition). The Jones representation $\rho$ is defined over integral Laurent polynomials $\mathbb{Z}\left[t, t^{-1}\right]$. The construction of $\rho$ is based on the Iwahori-Hecke algebra representations of Artin's braid group of 6 strings as well as the Birman-Hilden presentation of $\mathcal{M}_{2}$. The representation $\rho$ has been given attention as a candidate for the faithful linear representation of $\mathcal{M}_{2}$ ([3]). However, it seems that there are few results concerning $\rho$, with exceptions of a work of Humphries [8] and the author's previous result [10], both of which deal with specializations at roots of unity.

Let $\mathcal{I}_{2}$ denote the Torelli group of genus 2. It is defined as the kernel of the classical symplectic representation $\mathcal{M}_{2} \rightarrow S p(4, \mathbb{Z})$ which is induced by 
the natural action of $\mathcal{M}_{2}$ on the first integral homology group $H_{1}\left(\Sigma_{2} ; \mathbb{Z}\right)$ of $\Sigma_{2}$. Here, $S p(4, \mathbb{Z})$ denotes the Siegel modular group. In view of the explicit computation of $\rho$ given by Jones, it is easy to see that there are several special values of $t$ at which $\rho$ becomes trivial on $\mathcal{I}_{2}$. Such specializations include $t= \pm 1$. It is natural to consider perturbations of $t$ at such special values to extract any information of $\mathcal{I}_{2}$ in $\rho$.

In this paper, we consider the perturbation at $t=-1$. More precisely, let $\varphi$ be the expansion of $\rho$ obtained by setting $t=-e^{h}$ and then expanding the powers $e^{h}$ in their Taylor series. We then introduce a descending filtration of the Torelli group $\mathcal{I}_{2}$ by using $\varphi$, and present some results on the associated graded quotients. Our results should be considered as new properties of $\rho$. Our approach is based on Jones' explicit computation mentioned above.

Now we state our main results. We set

$\mathcal{F}_{k}=\mathcal{I}_{2} \cap\left\{f \in \mathcal{M}_{2} ; \varphi(f) \equiv\right.$ identity modulo terms of degrees higher than $\left.k\right\}$.

It will turn out that $\mathcal{F}_{1}$ coincides with $\mathcal{I}_{2}$ so that we have a filtration

$$
\mathcal{I}_{2}=\mathcal{F}_{1} \supset \mathcal{F}_{2} \supset \cdots \supset \mathcal{F}_{k} \supset \cdots .
$$

A general result by Lazard in [11] can be used to deduce that (a) for each $k \geq 1$, the graded quotient $\mathcal{G}_{k} \mathcal{F}=\mathcal{F}_{k} / \mathcal{F}_{k+1}$ is a free $\mathbb{Z}$-module of finite rank; (b) the filtration $\left\{\mathcal{F}_{k}\right\}$ is central so that the associated graded sum $\mathcal{G} \mathcal{F}=\oplus_{k \geq 1} \mathcal{G}_{k} \mathcal{F}$ naturally forms a graded Lie algebra over $\mathbb{Z}$. Furthermore, it turns out that $\mathcal{G}_{k} \mathcal{F}_{\mathbb{Q}}=\mathcal{G}_{k} \mathcal{F} \otimes \mathbb{Q}$ has a natural structure of a rational $S p(4, \mathbb{Q})$-module where $S p$ denotes the symplectic group. This enables us to use classical symplectic representation theory to study the structure of $\mathcal{G F}$.

Let $\Gamma_{a, b}$ denote the irreducible rational representation of $S p(4, \mathbb{Q})$ with highest weight $a L_{1}+b\left(L_{1}+L_{2}\right)$ (cf section 4.1). We understand that the same symbol also denotes the representation space over $\mathbb{Q}$ in the obvious manner. Our first result is:

Theorem 1.1 Let $k=1$. Then the following isomorphism of $\operatorname{Sp}(4, \mathbb{Q})$ modules holds:

$$
\mathcal{G}_{1} \mathcal{F}_{\mathbb{Q}}=\mathcal{I}_{2} / \mathcal{F}_{2} \otimes \mathbb{Q}=\Gamma_{0,2}
$$

The appearance of the module $\Gamma_{0,2}$ is suggestive since it also appears as the image of the second Johnson homomorphism $\tau_{2}(2)$ which is defined on $\mathcal{I}_{2}$ (see [16] as well as for the definition). In fact, we can show: 
Corollary 1.2 The kernel of the second Johnson homomorphism $\tau_{2}(2)$ coincides with $\mathcal{F}_{2}$. In particular, $\tau_{2}(2)$ factors through the Jones representation $\rho$.

Recall that Morita [13] has described the Casson invariant of homology 3spheres in terms of mapping class groups of surfaces. In particular, he has related the second Johnson homomorphism with the Casson invariant. In view of this, Corollary 1.2 raises a problem whether the Jones representation $\rho$ contains the full information of the Casson invariant in the case of genus 2. This problem will be reduced to the detection of a certain homomorphism of $\mathcal{I}_{2}$ which arises from Morita's theory of secondary characteristic classes of surface bundles [15], [16]. We will discuss this later in section 6 .

As for the module $\mathcal{G}_{k} \mathcal{F}_{\mathbb{Q}}$ for general $k$, we have the following.

Theorem 1.3 For each $k \geq 2$, the following holds:

(1) If $k$ is even, then $\mathcal{G}_{k} \mathcal{F}_{\mathbb{Q}} \supset \Gamma_{2,0}$.

(2) If $k$ is odd, then $\mathcal{G}_{k} \mathcal{F}_{\mathbb{Q}} \supset \Gamma_{0,2}$.

Our expansion $t=-e^{h}$ was motivated by the fact that a similar expansion decomposes the Jones polynomial of links, which can be also defined by the Iwahori-Hecke algebra representations of braid groups, into a series of Vassiliev invariants (see [2], [6], [4]). The reader may consider that an expansion $t=e^{h}$ would be more natural. In this case, however, the graded quotients associated with the induced filtration have structures of $S p(4, \mathbb{Z} / 2 \mathbb{Z})$-modules, rather than $S p(4, \mathbb{Q})$-modules. Thus the induced filtration does not seem to fit the context of the Torelli group. This phenomenon is due to the well known fact that the Iwahori-Hecke algebras (of type A) serve as deformations of group algebras of symmetric groups. We would like to study this aspect in detail elsewhere.

The organization of the present paper is as follows. In section 2, we recall some basic material concerned with the Jones representation $\rho$. We also determine the specialization of $\rho$ at $t=-1$, which will be the degree 0 part of the expanded representation $\varphi$. In section 3 , we describe the filtration $\left\{\mathcal{F}_{k}\right\}$ in a more convenient manner and establish its basic properties. In particular, we will see that each graded quotient $\mathcal{G}_{k} \mathcal{F}$ is naturally embedded into an $S p(4, \mathbb{Z})$-module which will be denoted by $\mathcal{G}_{k} R$. In section 4 , we show that $\mathcal{G}_{k} R$ is actually a rational $S p(4, \mathbb{Q})$-module, and then we apply the symplectic representation theory to decompose $\mathcal{G}_{k} R$ into weight spaces. In section 5 , we complete the proofs of our main results. Finally in section 6 , we discuss a possible relation with the Casson invariant. 


\section{Preliminaries}

\subsection{Jones' construction}

We start with recalling the general construction by Jones concerning $\rho$. We refer to [9] for further details. Let $\Sigma_{g}$ be a closed oriented surface of genus $g$ and let $\mathcal{M}_{g}$ be its mapping class group. The hyperelliptic mapping class group $\mathcal{H}_{g}$ is defined as the subgroup of $\mathcal{M}_{g}$ consisting of those elements which commute with the class of a fixed hyperelliptic involution. For $i=1,2, \ldots$, $(2 g+1)$, let $\zeta_{i}$ be the Dehn twist along the simple closed curve $C_{i}$ on $\Sigma_{g}$ depicted in Figure 1. Under an obvious choice of the hyperelliptic involution,

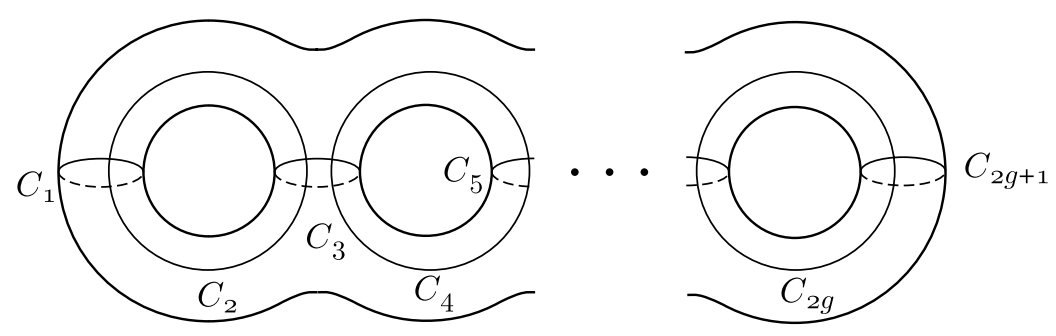

Figure 1

each $\zeta_{i}$ lies in $\mathcal{H}_{g}$. According to the presentation of $\mathcal{H}_{g}$ due to Birman and Hilden [5], $\mathcal{H}_{g}$ is generated by $\zeta_{1}, \zeta_{2}, \ldots, \zeta_{2 g+1}$, and these generators satisfy the defining relations of $B_{2 g+2}$, Artin's braid group of $(2 g+2)$ strings. We thus have a natural projection $p: B_{2 g+2} \rightarrow \mathcal{H}_{g}$. Recall that the irreducible representations of $B_{2 g+2}$ which arise from the Iwahori-Hecke algebra of type $A_{2 g+1}$ are in one-to-one correspondence with the Young diagrams of $(2 g+2)$ boxes [9]. Jones considered which of such representations of $B_{2 g+2}$ can yield a representation of $\mathcal{H}_{g}$ via $p$. Let $Y$ be a Young diagram of $(2 g+2)$ boxes, and let $\pi_{Y}$ denote the corresponding representation of $B_{2 g+2}$. Jones proved that $\pi_{Y}$ defines, via $p$, a projective linear representation of $\mathcal{H}_{g}$ if and only if $Y$ is rectangular. Furthermore, he proved that every projective representation of $\mathcal{H}_{g}$ so obtained lifts to an actual linear representation of $\mathcal{H}_{g}$ by determining the correcting scalar factor explicitly. Let us call this type of representation the Jones representation of genus $g$. For example the $1 \times(2 g+2)$ rectangular Young diagram gives the one dimensional trivial representation, and the $(2 g+2) \times 1$ one gives a one dimensional representation given by the correspondence $\zeta_{i} \mapsto$ $(-1)$ - identity. For later use, we denote the latter representation by sgn. We will also denote suitable scalar extensions of sgn by the same symbol. 


\subsection{The column-row symmetry}

For a Young diagram $Y$ with $(2 g+2)$ boxes, let $Y^{\prime}$ denote the Young diagram obtained by interchanging rows and columns in $Y$. There exists an algebra involution on the Iwahori-Hecke algebra of type $A_{2 g+1}$ which describes the transition from each $Y$ to $Y^{\prime}$ at the representation level (see [9, Note 4.6]). We can combine this involution with Jones' construction above to obtain the relation between the two Jones representations corresponding to rectangular Young diagrams $Y$ and $Y^{\prime}$, denoted by $\rho_{Y}$ and $\rho_{Y^{\prime}}$, respectively:

$$
\rho_{Y^{\prime}}\left(\zeta_{i}\right)=-\rho_{Y}\left(\zeta_{i}^{-1}\right)
$$

for $i=1, \ldots,(2 g+1)$. Note that the correspondence $\zeta_{i} \mapsto \zeta_{i}^{-1}$ defines an automorphism $\iota$ of $\mathcal{H}_{g}$, which can be realized by the conjugation by an orientation reversing involution on $\Sigma_{g}$. Thus we have an equality of representations:

$$
\rho_{Y^{\prime}}=\left(\rho_{Y} \circ \iota\right) \otimes \operatorname{sgn}
$$

for every rectangular Young diagram $Y$ with $(2 g+2)$ boxes.

\subsection{The genus 2 case}

In general, $\mathcal{H}_{g}$ is a proper subgroup of $\mathcal{M}_{g}$ and it is not obvious whether Jones representations of genus $g$ extend to $\mathcal{M}_{g}$ or not. However, in the case of $g=2$, it is classically known that $\mathcal{H}_{2}=\mathcal{M}_{2}$ so that all the Jones representations of genus 2 are defined on $\mathcal{M}_{2}$ for a trivial reason. Furthermore, in view of (2.1), the non-trivial Jones representation of genus 2 is essentially unique. This unique representation, which corresponds to the $3 \times 2$ rectangular Young diagram, is the main object of this paper, and is denoted by $\rho$.

As was mentioned above, $\mathcal{M}_{2}$ is generated by $\zeta_{1}, \ldots, \zeta_{5}$. Jones has explicitly computed the images of these generators under $\rho$, which can be taken as the definition of $\rho$. Instead of $\zeta_{i}$ 's, we use another set of generators of $\mathcal{M}_{2}$ which is more convenient for our computation. Let us take an element $\xi=\zeta_{1} \zeta_{2} \cdots \zeta_{5} \in \mathcal{M}_{2}$ which is a periodic automorphism of order 6 . It is easy to see that $\zeta_{i+1}=\xi \zeta_{i} \xi^{-1}$ for $i=1,2, \ldots, 4$. Thus we can choose $\zeta_{1}$ and $\xi$ to be generators of $\mathcal{M}_{2}$. Now, due to the computation by Jones, the Jones representation $\rho: \mathcal{M}_{2} \rightarrow G L\left(5, \mathbb{Z}\left[t, t^{-1}\right]\right)$ can be defined by the following:

$$
\rho\left(\zeta_{1}\right)=\left(\begin{array}{ccccc}
-1 / t^{2} & 0 & 0 & 0 & t^{3} \\
0 & -1 / t^{2} & 1 / t^{2} & 0 & 0 \\
0 & 0 & t^{3} & 0 & 0 \\
0 & 0 & 1 / t^{2} & -1 / t^{2} & 0 \\
0 & 0 & 0 & 0 & t^{3}
\end{array}\right),
$$


and

$$
\rho(\xi)=\left(\begin{array}{ccccc}
0 & 0 & 1 & 0 & 0 \\
0 & 0 & 0 & 0 & 1 \\
1 & 0 & 0 & 0 & 0 \\
0 & 1 & 0 & 0 & 0 \\
0 & 0 & 0 & 1 & 0
\end{array}\right)
$$

Here our parameter $t$ corresponds to the formal power $q^{1 / 5}$ in [9].

Recall that the Torelli group of genus 2, denoted by $\mathcal{I}_{2}$, is the kernel of the symplectic representation $\mathcal{M}_{2} \rightarrow S p(4, \mathbb{Z})$. Due to Powell [17], $\mathcal{I}_{2}$ is the normal closure in $\mathcal{M}_{2}$ of the single Dehn twist $\psi_{0}$ along the separating simple closed curve $C_{0}$ on $\Sigma_{2}$ depicted in Figure 2. The image of $\psi_{0}$ under $\rho$ has also been

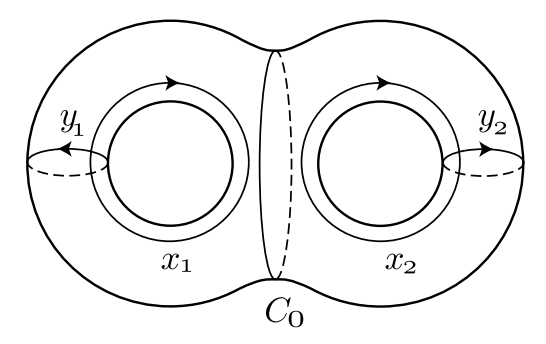

Figure 2

computed by Jones. In our notation,

$$
\begin{aligned}
\rho\left(\psi_{0}\right) & =\rho\left(\zeta_{1} \zeta_{2} \zeta_{1}\right)^{4}=t^{6} \cdot I d \\
& +\frac{t^{15}+1}{t^{24}} \cdot\left(\begin{array}{ccccc}
0 & 0 & 0 & 0 & 0 \\
0 & 0 & 0 & 0 & 0 \\
0 & 0 & 0 & 0 & 0 \\
t^{10}-1 & -t^{10}+t^{5} & t^{10}-1 & -t^{15}+1 & -t^{10}+t^{5} \\
0 & 0 & 0 & 0 & 0
\end{array}\right)
\end{aligned}
$$

where $I d$ denotes the identity matrix. In particular, this shows that $\rho$ does not factor through the symplectic representation. On the other hand, it is easy to see that there exist several special values of $\rho$ at which $\rho$ becomes trivial on $\mathcal{I}_{2}$. Such special values include $t= \pm 1$.

We now determine the specialization of $\rho$ at $t=-1$. This will play a fundamental role in our investigation as the degree 0 part of our expansion of $\rho$. Let $H$ denote the first integral homology group $H_{1}\left(\Sigma_{2} ; \mathbb{Z}\right)$ of $\Sigma_{2}$, and let $\Lambda^{2} H$ denote the second exterior product of $H$. The symplectic class $\omega \in \Lambda^{2} H$ is 
defined as $x_{1} \wedge y_{1}+x_{2} \wedge y_{2}$ for any symplectic basis $x_{i}, y_{i}(i=1,2)$ of $H$ with respect to the algebraic intersection pairing.

Lemma 2.1 The specialization of $\rho$ at $t=-1$ descends to a linear representation of $S p(4, \mathbb{Z})$ via the symplectic representation. Furthermore, this specialization is equivalent to $\left(\Lambda^{2} H / \omega \cdot \mathbb{Z}\right) \otimes \operatorname{sgn}$.

For later use, we fix a symplectic basis of $H$ and a basis of $\Lambda^{2} H / \omega \cdot \mathbb{Z}$. Let $x_{1}, x_{2}, y_{1}, y_{2}$ be the symplectic basis of $H$ depicted in Figure 2 . We choose a basis $\left\{t_{i} ; i=1, \ldots, 5\right\}$ of $\Lambda^{2} H / \omega \cdot \mathbb{Z}$ as follows:

$$
\begin{aligned}
& t_{1}=\left[x_{1} \wedge x_{2}\right], \quad t_{2}=\left[y_{1} \wedge y_{2}\right], \quad t_{3}=\left[x_{1} \wedge y_{1}\right], \\
& t_{4}=\left[x_{1} \wedge y_{2}\right], \quad t_{5}=\left[x_{2} \wedge y_{1}\right]
\end{aligned}
$$

where [ ] denotes the corresponding class in $\Lambda^{2} H / \omega \mathbb{Z}$.

Proof of Lemma 2.1 As was mentioned above, the former part of the lemma is a direct consequence of (2.2). For the latter part, let $P$ denote the specialization of $\rho$ obtained by putting $t=-1$. Note that $P$ is defined over $\mathbb{Z}$ so that its representation space is $\mathbb{Z}^{5}$. Let $Z$ and $X$ denote the matrix form of the action of $\zeta_{1}$ and $\xi$, respectively, on $\Lambda^{2} H / \omega \mathbb{Z}$ with respect to the basis $\left\{t_{i}\right\}$ above. Then the assertion follows from the existence of $F \in G L(5, \mathbb{Z})$ such that $P\left(\zeta_{1}\right) F=-F Z$ and $P(\xi) F=-F X$. A direct calculation shows that the following matrix gives a solution:

$$
F=\left(\begin{array}{ccccc}
0 & -1 & 0 & 0 & 0 \\
0 & 0 & 0 & 0 & -1 \\
1 & 0 & 0 & 0 & 0 \\
0 & 0 & 1 & 1 & -1 \\
0 & 0 & 0 & 1 & 0
\end{array}\right)
$$

\section{The filtration $\left\{\mathcal{F}_{k}\right\}$}

\subsection{The description of $\mathcal{F}_{k}$}

As in Introduction, we obtain the representation

$$
\varphi: \mathcal{M}_{2} \rightarrow R^{\times}
$$

from $\rho$ by putting $t=-e^{h}$ and then expanding the powers $e^{h}$ in their Taylor series. Here $R$ denotes the algebra $M(5, \mathbb{Q}[[h]])$ of $5 \times 5$ matrices over rational 
formal power series in a variable $h$, and ${ }^{x}$ denotes the group of invertible elements of an algebra. Hereafter, the identity of an algebra will be denoted by 1.

For $k \geq 0$, let $R[k]$ be the principal ideal of $R$ generated by $h^{k} \cdot 1$. The ideal $R[k]$ consists of those elements in $R$ the minimal degrees of whose terms are at least $k$. Thus we have a filtration of $R$ :

$$
R=R[0] \supset R[1] \supset R[2] \supset \cdots
$$

Note that $R[k] \cdot R[l]=R[k+l]$. Let $\mathcal{G}_{k} R$ be the graded quotient $R[k] / R[k+1]$ and let $\mathcal{G} R=\oplus_{k \geq 0} \mathcal{G}_{k} R$ be the associated graded algebra. The standard bracket $[X, Y]=X Y-Y X$ in $R$ induces a structure of a graded Lie algebra over $\mathbb{Q}$ on $\mathcal{G} R$.

Let $\varphi^{(k)}: \mathcal{M}_{2} \rightarrow(R / R[k+1])^{\times}$be the composition of $\varphi$ with the natural projection $R^{\times} \rightarrow(R / R[k+1])^{\times}$. Now the filtration $\left\{\mathcal{F}_{k}\right\}$ can be described as $\mathcal{F}_{k}=\mathcal{I}_{2} \cap \operatorname{Ker} \varphi^{(k-1)}$ for $k \geq 1$. Obviously, $\varphi^{(0)}$ is equivalent to the specialization of $\rho$ at $t=-1$. Thus we have $\mathcal{F}_{1}=\mathcal{I}_{2}$ by Lemma 2.1. As before, we write $\mathcal{G}_{k} \mathcal{F}$ for $\mathcal{F}_{k} / \mathcal{F}_{k+1}$, and $\mathcal{G} \mathcal{F}$ for $\oplus_{k \geq 1} \mathcal{G}_{k} \mathcal{F}$.

\subsection{The graded Lie algebra $\mathcal{G} \mathcal{F}$}

We define a mapping $\delta: \mathcal{I}_{2} \rightarrow R$ by $\delta(\psi)=\varphi(\psi)-1$. It is obvious that $\delta\left(\mathcal{F}_{k}\right) \subset R[k]$. For each $k \geq 1$, let $\delta_{k}$ be the composition of $\delta$ restricted to $\mathcal{F}_{k}$ with the obvious projection $R[k] \rightarrow \mathcal{G}_{k} R$. The following proposition can be deduced from a general result of Lazard [11] (see also [12]).

Proposition 3.1 Each $\delta_{k}$ induces an embedding of the module $\mathcal{G}_{k} \mathcal{F}$ into $\mathcal{G}_{k} R$. Furthermore, the commutator operation $[x, y]=x y x^{-1} y^{-1}$ induces a structure of a graded Lie algebra on $\mathcal{G} \mathcal{F}$, and $\oplus \delta_{k}$ gives an embedding of a graded Lie algebra $\mathcal{G F} \rightarrow \mathcal{G} R$.

Remark 3.2 The quotient module $\mathcal{G}_{k} R$ has a natural structure of a finite dimensional vector space over $\mathbb{Q}$. Thus the proposition implies that each $\mathcal{G}_{k} \mathcal{F}$ is a free abelian group of finite rank.

We give a direct proof of Proposition 3.1 for completeness.

Lemma 3.3 For $k \geq 1$, the mapping $\delta_{k}: \mathcal{F}_{k} \rightarrow \mathcal{G}_{k} R=R[k] / R[k+1]$ is a group homomorphism. 
Proof If $\psi_{1}, \psi_{2} \in \mathcal{F}_{k}$, then we have $\delta\left(\psi_{1}\right) \delta\left(\psi_{2}\right) \in R[2 k] \subset R[k+1]$. Thus the lemma follows immediately from the following equality:

$$
\delta\left(\psi_{1} \psi_{2}\right)=\delta\left(\psi_{1}\right)+\delta\left(\psi_{2}\right)+\delta\left(\psi_{1}\right) \delta\left(\psi_{2}\right) .
$$

Clearly $\operatorname{Ker} \delta_{k}=\mathcal{F}_{k+1}$. Thus $\delta_{k}$ gives an embedding of a group

$$
\mathcal{G}_{k} \mathcal{F} \rightarrow \mathcal{G}_{k} R
$$

which will be denoted by the same symbol $\delta_{k}$.

Now let $\mathcal{G} \delta$ denote $\oplus_{k \geq 1} \delta_{k}: \mathcal{G F} \rightarrow \mathcal{G} R$. To prove the latter part of the proposition, we have to see that $\operatorname{Im} \mathcal{G} \delta$ is a Lie subalgebra of $\mathcal{G} R$, and that the Lie bracket on $\mathcal{G F}$ induced by $\mathcal{G} \delta$ corresponds to the commutator in $\mathcal{I}_{2}$. These follow from the next lemma.

Lemma 3.4 Let $x \in \mathcal{F}_{k}$ and $y \in \mathcal{F}_{l}$. Then $[x, y] \in \mathcal{F}_{k+l}$, and the following equality holds:

$$
\delta_{k+l}([x, y])=\delta_{k}(x) \delta_{l}(y)-\delta_{l}(y) \delta_{k}(x)
$$

Proof A direct computation implies the following equality in $R$ :

$$
\delta([x, y])=(\delta(x) \delta(y)-\delta(y) \delta(x)) \varphi\left(x^{-1} y^{-1}\right)
$$

This implies immediately that $[x, y] \in \mathcal{F}_{k+l}$. Furthermore, in view of the fact that $\varphi^{(0)}$, the degree 0 part of $\varphi$, is trivial on $\mathcal{I}_{2}$ (Lemma 2.1), we obtain the required equality by taking (3.1) modulo $\mathcal{F}_{k+l+1}$.

This completes the proof of Proposition 3.1.

\section{3 $S p(4, \mathbb{Z})$-actions}

For each $k, \mathcal{G}_{k} \mathcal{F}$ naturally has a structure of an $S p(4, \mathbb{Z})$-module in the following manner. The Torelli group $\mathcal{I}_{2}=\mathcal{F}_{1}$ is acted on by $\mathcal{M}_{2}$ via conjugation. The filtration $\left\{\mathcal{F}_{k}\right\}$ is preserved by this action of $\mathcal{M}_{2}$ so that $\mathcal{G}_{k} \mathcal{F}$ is an $\mathcal{M}_{2}-$ module. Since $\left[\mathcal{I}_{2}, \mathcal{F}_{k}\right] \subset \mathcal{F}_{k+1}$ by Lemma 3.4, the action of $\mathcal{I}_{2}$ on $\mathcal{G}_{k} \mathcal{F}$ is trivial. Hence the above action of $\mathcal{M}_{2}$ descends to that of $\operatorname{Sp}(4, \mathbb{Z})$.

Next we consider the natural action of $S p(4, \mathbb{Z})$ on $\mathcal{G}_{k} R$ for each $k \geq 0$. Recall that $\varphi^{(k)}$ denotes the composition of the expanded Jones representation $\varphi$ with the projection $R^{\times} \rightarrow(R / R[k+1])^{\times}$. For each $\zeta \in \mathcal{M}_{2}$, the correspondence $x \mapsto$ $\zeta_{*} x=\varphi^{(k)}(\zeta) x \varphi^{(k)}\left(\zeta^{-1}\right)$ induces a structure of an $\mathcal{M}_{2}$-module on $R / R[k+1]$. 
Thus $\mathcal{G}_{k} R \subset R / R[k+1]$ is an $\mathcal{M}_{2}$-submodule. It is easy to see that the following formula holds

$$
\zeta_{*} x=\varphi^{(0)}(\zeta) \cdot x \cdot \varphi^{(0)}\left(\zeta^{-1}\right)
$$

for $\zeta \in \mathcal{M}_{2}$ and $x \in \mathcal{G}_{k} R$. Here the multiplication in the right hand side is meant by the one in the graded algebra $\mathcal{G} R$. Now the triviality of $\varphi^{(0)}$ on $\mathcal{I}_{2}$ (Lemma 2.1) implies that the action of $\mathcal{M}_{2}$ on $\mathcal{G}_{k} R$ factors through $S p(4, \mathbb{Z})$.

Proposition 3.5 The embedding of Lie algebra $\mathcal{G} \delta: \mathcal{G F} \rightarrow \mathcal{G} R$ is $S p(4, \mathbb{Z})$ equivariant.

Proof It suffices to show that $\delta_{k}\left(\zeta \psi \zeta^{-1}\right)=\zeta_{*} \delta_{k}(\psi)$ for $\zeta \in \mathcal{M}_{2}, \psi \in \mathcal{F}_{k}$. By definition, we have $\delta\left(\zeta \psi \zeta^{-1}\right)=\varphi(\zeta) \delta(\psi) \varphi\left(\zeta^{-1}\right)$. Thus taking the $\bmod R[k+1]$ classes, we have $\delta_{k}\left(\zeta \psi \zeta^{-1}\right)=\varphi^{(0)}(\zeta) \cdot \delta_{k}(\psi) \cdot \varphi^{(0)}\left(\zeta^{-1}\right)$ as elements of $\mathcal{G} R$. In view of $(3.2)$, this is nothing but $\zeta_{*} \delta_{k}(\psi)$.

\section{The structure of $\mathcal{G}_{k} R$}

\subsection{Irreducible representations of $S p(4, \mathbb{Q})$}

Now we recall the description of irreducible representations of the algebraic group $S p(4, \mathbb{Q})$. We follow the book of Fulton and Harris [7]. Let $\mathfrak{s p}(4, \mathbb{C})$ be the Lie algebra of the symplectic Lie group $S p(4, \mathbb{C})$, and let $\mathfrak{h}$ be its Cartan subalgebra consisting of diagonal matrices. Choose a system of fundamental weights $L_{1}$ and $L_{2}: \mathfrak{h} \rightarrow \mathbb{C}$ as in [7]. Then for each pair $(a, b)$ of non-negative integers, there exists a unique irreducible representation of $S p(4, \mathbb{C})$ with highest weight $a L_{1}+b\left(L_{1}+L_{2}\right)$. We denote this representation by $\Gamma_{a, b}$ following [7]. These are all rational representations defined over $\mathbb{Q}$ so that we can consider them as irreducible representations of $S p(4, \mathbb{Q})$. We will understand that the same notation $\Gamma_{a, b}$ also denotes the representation space over $\mathbb{Q}$ of the corresponding representation in the obvious manner. For example the trivial representation $\mathbb{Q}=\Gamma_{0,0}, H_{\mathbb{Q}}=H_{1}\left(\Sigma_{2} ; \mathbb{Q}\right)=\Gamma_{1,0}$ and $\Lambda^{2} H_{\mathbb{Q}} / \omega \mathbb{Q}=\Gamma_{0,1}$ where $\omega$ denotes the symplectic class.

\subsection{The action of $S p(4, \mathbb{Q})$ on $\mathcal{G}_{k} R$}

For an $S p(4, \mathbb{Z})$-module $V$, let $V_{\mathbb{Q}}$ denote $V \otimes \mathbb{Q}$. In view of $(3.2)$, it is easy to see that the $S p(4, \mathbb{Z})$-module $\mathcal{G}_{k} R$ is isomorphic to $\left(\varphi^{(0)} \otimes\left(\varphi^{(0)}\right)^{*}\right)_{\mathbb{Q}}$ where * 
denotes the dual of a representation. By Lemma 2.1, we have $\varphi_{\mathbb{Q}}^{(0)}=\Gamma_{0,1} \otimes \operatorname{sgn}$, which is only an $S p(4, \mathbb{Z})$-module. However, the triviality of $\operatorname{sgn} \otimes \operatorname{sgn}^{*}=$ $\operatorname{sgn} \otimes \operatorname{sgn}$ implies that

$$
\left(\varphi^{(0)} \otimes\left(\varphi^{(0)}\right)^{*}\right)_{\mathbb{Q}}=\Gamma_{0,1} \otimes \Gamma_{0,1}^{*}=\Gamma_{0,1} \otimes \Gamma_{0,1}
$$

so that $\mathcal{G}_{k} R$ can be naturally considered as an $S p(4, \mathbb{Q})$-module. Here we have used the self-duality for general symplectic modules to deduce the last equality. Furthermore, the decomposition of $\Gamma_{0,1} \otimes \Gamma_{0,1}$ into irreducible modules is well known (eg [7, section 16.2]), which implies the equality

$$
\mathcal{G}_{k} R=\Gamma_{0,2} \oplus \Gamma_{2,0} \oplus \Gamma_{0,0} .
$$

\subsection{Weight spaces of $\mathcal{G}_{k} R$}

Now we explicitly describe the decomposition of $\mathcal{G}_{k} R=\Gamma_{0,1} \otimes \Gamma_{0,1}^{*}$ into weight spaces and determine the submodule $\Gamma_{0,2}$ in terms of weight spaces. Recall that we have fixed the basis $\left\{t_{i} ; i=1, \ldots, 5\right\}$ of $\Lambda^{2} H / \omega \mathbb{Z}$ in $(2.3)$. The $\left\{t_{i}\right\}$ also serves as a basis of $\Gamma_{0,1}=\left(\Lambda^{2} H / \omega \mathbb{Z}\right) \otimes \mathbb{Q}$ in the obvious manner. All the members $t_{i}$ 's are weight vectors in $\Gamma_{0,1}$ and the corresponding weights are: $\left(L_{1}+L_{2}\right),-\left(L_{1}+L_{2}\right), 0,\left(L_{1}-L_{2}\right)$ and $\left(-L_{1}+L_{2}\right)$ for $i=1, \ldots, 5$, respectively. These can be deduced from the description of the weight spaces for the fundamental representation $H_{\mathbb{Q}}=H_{1}\left(\Sigma_{2} ; \mathbb{Q}\right)$.

Now let $\left\{t_{i}^{*}\right\}$ denote the dual basis of $\left\{t_{i}\right\}$, and let $e_{i, j}$ denote $t_{i} \otimes t_{j}^{*} \in \Gamma_{0,1} \otimes \Gamma_{0,1}^{*}$. Then $\left\{e_{i, j}\right\}$ forms a basis of $\Gamma_{0,1} \otimes \Gamma_{0,1}^{*}$. Furthermore, each $e_{i, j}$ is a weight vector and its weight is given by the weight of $t_{i}$ minus that of $t_{j}$. Consequently, we can obtain the decomposition of $\Gamma_{0,1} \otimes \Gamma_{0,1}^{*}$ into weight spaces as given by Table 1. Each row of Table 1 consists of a weight appearing in $\Gamma_{0,1} \otimes \Gamma_{0,1}^{*}$ and a basis for the corresponding weight space. The identification of $\Gamma_{0,1} \otimes \Gamma_{0,1}^{*}=\operatorname{End}\left(\Gamma_{0,1}\right)$ with $\mathcal{G}_{k} R$ is given as follows. Let $E_{i, j} \in M(5, \mathbb{Q})$ denote the matrix form of $e_{i, j} \in \operatorname{End}\left(\Gamma_{0,1}\right)$ with respect to the basis $\left\{t_{i}\right\}$. As was observed in the proof of Lemma 2.1, $\Gamma_{0,1}$ is identified with $\varphi^{(0)} \otimes \operatorname{sgn}$ via the matrix $F$ given by (2.4). Then the desired identification is given by the correspondence

$$
e_{i, j} \mapsto h^{k} F E_{i, j} F^{-1} \bmod R[k+1] .
$$

Next we describe the weight spaces of $\Gamma_{0,2}$. Note that $\Gamma_{0,2}$ is the highest weight submodule of $\Gamma_{0,1} \otimes \Gamma_{0,1}^{*}$. Recall from the general representation theory that the highest weight submodule is generated by the images of a highest weight vector, which are also weight vectors, under successive applications of the negative root spaces of the Lie algebra. Due to this fact, we can obtain the weight spaces of $\Gamma_{0,2}$ starting with a highest weight vector $e_{1,2}$. The result is given by Table 2 . 
Table 1: The weight spaces of $\Gamma_{0,1} \otimes \Gamma_{0,1}^{*}$

\begin{tabular}{r|l}
\hline Weight & A basis for the weight space \\
\hline $2\left(L_{1}+L_{2}\right)$ & $\left\{e_{1,2}\right\}$ \\
$L_{1}+L_{2}$ & $\left\{e_{1,3}, e_{3,2}\right\}$ \\
$2 L_{1}$ & $\left\{e_{1,5}, e_{4,2}\right\}$ \\
$2 L_{2}$ & $\left\{e_{1,4}, e_{5,2}\right\}$ \\
$2\left(L_{1}-L_{2}\right)$ & $\left\{e_{4,5}\right\}$ \\
$L_{1}-L_{2}$ & $\left\{e_{3,5}, e_{4,3}\right\}$ \\
0 & $\left\{e_{i, i} ; i=1, \ldots, 5\right\}$ \\
$-L_{1}+L_{2}$ & $\left\{e_{3,4}, e_{5,3}\right\}$ \\
$2\left(-L_{1}+L_{2}\right)$ & $\left\{e_{5,4}\right\}$ \\
$-2 L_{2}$ & $\left\{e_{2,5}, e_{4,1}\right\}$ \\
$-2 L_{1}$ & $\left\{e_{2,4}, e_{5,1}\right\}$ \\
$-\left(L_{1}+L_{2}\right)$ & $\left\{e_{2,3}, e_{3,1}\right\}$ \\
$-2\left(L_{1}+L_{2}\right)$ & $\left\{e_{2,1}\right\}$ \\
\hline
\end{tabular}

Table 2: The weight spaces of $\Gamma_{0,2}$

\begin{tabular}{r|l}
\hline Weight & A basis for the weight space \\
\hline $2\left(L_{1}+L_{2}\right)$ & $\left\{e_{1,2}\right\}$ \\
$L_{1}+L_{2}$ & $\left\{e_{1,3}+2 e_{3,2}\right\}$ \\
$2 L_{1}$ & $\left\{e_{1,5}+e_{4,2}\right\}$ \\
$2 L_{2}$ & $\left\{e_{1,4}+e_{5,2}\right\}$ \\
$2\left(L_{1}-L_{2}\right)$ & $\left\{e_{4,5}\right\}$ \\
$L_{1}-L_{2}$ & $\left\{e_{4,3}+2 e_{3,5}\right\}$ \\
0 & $\left\{\left(e_{1,1}+e_{2,2}\right)-\left(e_{4,4}+e_{5,5}\right), 2 e_{3,3}-\left(e_{1,1}+e_{2,2}\right)\right\}$ \\
$L_{2}-L_{1}$ & $\left\{2 e_{3,4}+e_{5,3}\right\}$ \\
$2\left(L_{2}-L_{1}\right)$ & $\left\{e_{5,4}\right\}$ \\
$-2 L_{2}$ & $\left\{e_{2,5}+e_{4,1}\right\}$ \\
$-2 L_{1}$ & $\left\{e_{2,4}+e_{5,1}\right\}$ \\
$-\left(L_{1}+L_{2}\right)$ & $\left\{e_{2,3}+2 e_{3,1}\right\}$ \\
$-2\left(L_{1}+L_{2}\right)$ & $\left\{e_{2,1}\right\}$ \\
\hline
\end{tabular}

\section{Proofs of Theorems}

First of all, by virtue of the following general proposition, the action of $S p(4, \mathbb{Z})$ on $\mathcal{G}_{k} \mathcal{F}$ described in section 3.3 can be extended to an action of $S p(4, \mathbb{Q})$ on $\mathcal{G}_{k} \mathcal{F}_{\mathbb{Q}}=\mathcal{G}_{k} \mathcal{F} \otimes \mathbb{Q}$ so that $\mathcal{G} \delta \otimes \mathbb{Q}$ is an $S p(4, \mathbb{Q})$-equivariant homomorphism between graded Lie algebras over $\mathbb{Q}$.

Algebraic 83 Geometric Topology, Volume 1 (2001) 
Proposition 5.1 (Asada-Nakamura [1, (2.2.8)]) Let $g \geq 1$. If $L$ is a $\mathbb{Z}$ submodule of a rational finite dimensional $S p(2 g, \mathbb{Q})$-module $V$ which is stable under the action of $S p(2 g, \mathbb{Z})$, then $L \otimes \mathbb{Q} \subset V$ is also stable under the action of $\operatorname{Sp}(2 g, \mathbb{Q})$.

\subsection{Proof of Theorem 1.1}

In view of (4.1), it suffices to show that $\delta_{1}\left(\mathcal{F}_{1}\right)_{\mathbb{Q}}=\Gamma_{0,2} \subset \mathcal{G}_{1} R$. As was explained in section $2.3, \mathcal{F}_{1}=\mathcal{I}_{2}$ is the normal closure of $\psi_{0}$, the Dehn twist along the separating simple closed curve $C_{0}$. Thus $\delta_{1}\left(\mathcal{F}_{1}\right)$ is the minimal $\mathbb{Z}_{-}^{-}$ submodule of $\mathcal{G}_{1} R$ which contains $\delta_{1}\left(\psi_{0}\right)$ and is invariant under the action of $S p(4, \mathbb{Z})$. A direct computation shows that

$$
\delta_{1}\left(\psi_{0}\right) \equiv F \cdot\left(\begin{array}{ccccc}
6 h & 0 & 0 & 0 & 0 \\
0 & 6 h & 0 & 0 & 0 \\
0 & 0 & -24 h & 0 & 0 \\
0 & 0 & 0 & 6 h & 0 \\
0 & 0 & 0 & 0 & 6 h
\end{array}\right) \cdot F^{-1} \bmod R[2]
$$

where the matrix $F$ is the one given in (2.4). This corresponds, under the identification (4.2), to

$$
-12\left(2 e_{3,3}-\left(e_{1,1}+e_{2,2}\right)\right)-6\left(\left(e_{1,1}+e_{2,2}\right)-\left(e_{4,4}+e_{5,5}\right)\right) \in \Gamma_{0,1} \otimes \Gamma_{0,1}^{*} .
$$

In view of Table 2 , this is a weight vector of $\Gamma_{0,2} \subset \Gamma_{0,1} \otimes \Gamma_{0,1}^{*}$ with weight 0 . Thus we have $\delta_{1}\left(\mathcal{F}_{1}\right) \subset \Gamma_{0,2}$. Now we can apply Proposition 5.1 to conclude that $\delta_{1}\left(\mathcal{F}_{1}\right)_{\mathbb{Q}}=\Gamma_{0,2}$.

\subsection{Proof of Corollary 1.2}

We freely use the notation of [16, section 5] for Johnson homomorphisms. To prove Corollary 1.2 , we have only to prove that there exists an $S p(4, \mathbb{Q}$ )equivariant isomorphism $f: \delta_{1}\left(\mathcal{I}_{2}\right)_{\mathbb{Q}} \rightarrow \tau_{2}(2)\left(\mathcal{I}_{2}\right)_{\mathbb{Q}}=\tau_{2}(2)\left(\mathcal{I}_{2}\right) \otimes \mathbb{Q}$ such that

$$
f \circ \delta_{1}\left(\psi_{0}\right)=\tau_{2}(2)\left(\psi_{0}\right) \text {. }
$$

As mentioned in Introduction, it is well known that $\tau_{2}(2)\left(\mathcal{I}_{2}\right)_{\mathbb{Q}}$ is isomorphic to $\Gamma_{0,2}$, and hence there exists an essentially unique $S p(4, \mathbb{Q})$-isomorphism between $\delta_{1}\left(\mathcal{I}_{2}\right)_{\mathbb{Q}}$ and $\tau_{2}(2)\left(\mathcal{I}_{2}\right)_{\mathbb{Q}}$. However, the existence of $f$ satisfying the condition (5.1) does not follow from this fact directly. We need an analysis of the images of $\psi_{0}$ under both $\delta_{1}$ and $\tau_{2}(2)$ in terms of highest weight vectors. 
Recall that $\delta_{1}\left(\mathcal{I}_{2}\right)_{\mathbb{Q}}$ is generated by the images of its highest weight vector under the action of the universal enveloping algebra $U$ of $\mathfrak{s p}(4, \mathbb{Q})$. Thus $\delta_{1}\left(\psi_{0}\right)$ can be expressed as $Z \cdot e_{1,2}$ for some fixed element $Z \in U$.

On the other hand, an explicit computation of $\tau_{g, 1}(2)$, a relative version of $\tau_{g}(2)$, has been given by Morita [13]. We can combine this result with the relationship between $\tau_{g, 1}(2)$ and $\tau_{g}(2)$ explained in [16] to obtain the description of $\tau_{g}(2)$. More precisely, we can describe (a) the decomposition of $\tau_{2}(2)\left(\mathcal{I}_{2}\right)_{\mathbb{Q}}$ into weight spaces together with the action of $\mathfrak{s p}(4, \mathbb{Q}) ;\left(\right.$ b) an expression of $\tau_{2}(2)\left(\psi_{0}\right)$ in terms of weight vectors. As a result, we can choose a highest weight vector $v_{0}$ of $\tau_{2}(2)\left(\mathcal{I}_{2}\right)_{\mathbb{Q}}$ such that $\tau_{2}(2)\left(\psi_{0}\right)=Z \cdot v_{0}$. If we take $f$ to be the unique $S p(4, \mathbb{Q})$-equivariant isomorphism which sends $e_{1,2}$ to $v_{0}$, then it satisfies the condition (5.1). This completes the proof of Corollary 1.2 .

\subsection{Proof of Theorem $\mathbf{1 . 3}$}

We show that the desired submodules come from the lower central series of the Torelli group $\{\mathcal{I}[k]\}$ where $\mathcal{I}[1]=\mathcal{I}_{2}$ and $\mathcal{I}[k+1]$ is defined recursively by $\mathcal{I}[k+$ $1]=\left[\mathcal{I}_{2}, \mathcal{I}[k]\right]$ for $k \geq 1$. More precisely, let $\mathcal{G D}_{\mathbb{Q}}$ denote the Lie subalgebra of $\mathcal{G F}_{\mathbb{Q}}$ generated by $\mathcal{G}_{1} \mathcal{F}_{\mathbb{Q}}$. We write $\mathcal{G}_{k} \mathcal{D}_{\mathbb{Q}}$ for its homogeneous component of degree $k$ so that we have $\mathcal{G}_{1} \mathcal{D}_{\mathbb{Q}}=\mathcal{G}_{1} \mathcal{F}_{\mathbb{Q}}$ and $\mathcal{G}_{k+1} \mathcal{D}_{\mathbb{Q}}=\left[\mathcal{G}_{1} \mathcal{D}_{\mathbb{Q}}, \mathcal{G}_{k} \mathcal{D}_{\mathbb{Q}}\right] \subset$ $\mathcal{G}_{k+1} \mathcal{F}_{\mathbb{Q}}$ for $k \geq 1$. It is obvious that $\mathcal{G}_{k} \mathcal{D}_{\mathbb{Q}}=\delta_{k}(\mathcal{I}[k] / \mathcal{I}[k+1]) \otimes \mathbb{Q}$. We assert that

$$
\mathcal{G}_{k} \mathcal{D}_{\mathbb{Q}}=\left\{\begin{array}{l}
\Gamma_{2,0} \text { for } k \text { even } \\
\Gamma_{0,2} \text { for } k \text { odd }
\end{array}\right.
$$

as a submodule of End $\left(\Gamma_{0,1}\right)$ under the identification of (4.2). Under the same identification, the Lie bracket $[]:, \mathcal{G}_{k} R \otimes \mathcal{G}_{l} R \rightarrow \mathcal{G}_{k+l} R$ corresponds to the standard Lie bracket in End $\left(\Gamma_{0,1}\right)$ given by $[A, B]=A B-B A$. Since we have already seen that $\mathcal{G}_{1} \mathcal{D}_{\mathbb{Q}}=\Gamma_{0,2}$, the assertion above follows from the next two equalities in End $\left(\Gamma_{0,1}\right)$ :

$$
\begin{aligned}
& {\left[\Gamma_{0,2}, \Gamma_{0,2}\right]=\Gamma_{2,0}} \\
& {\left[\Gamma_{0,2}, \Gamma_{2,0}\right]=\Gamma_{0,2}}
\end{aligned}
$$

These equalities can be checked as follows. First we can easily see that the Lie bracket is $S p(4, \mathbb{Q})$-equivariant so that both the left hand sides above are direct sums of some of $\Gamma_{0,2}, \Gamma_{2,0}$ and $\Gamma_{0,0}$.

Next, we can use Table 2 to compute the dimensions of weight spaces in $\left[\Gamma_{0,2}, \Gamma_{0,2}\right]$ with weights $2\left(L_{1}+L_{2}\right), 2 L_{1}$ and 0 , respectively. The results are 0,1 and 2 , respectively. Thus the highest weight of $\left[\Gamma_{0,2}, \Gamma_{0,2}\right]$ is $2 L_{1}$, which 
implies that $\Gamma_{0,2} \not \subset\left[\Gamma_{0,2}, \Gamma_{0,2}\right]$ and $\Gamma_{2,0} \subset\left[\Gamma_{0,2}, \Gamma_{0,2}\right]$. Finally we can see that $\Gamma_{0,0} \not \subset\left[\Gamma_{0,2}, \Gamma_{0,2}\right]$ by comparing the dimension of the weight space of weight 0 in $\left[\Gamma_{0,2}, \Gamma_{0,2}\right]$ with that in $\Gamma_{2,0}(\mathrm{cf}[7])$. This concludes the first equality.

Now with the assistance of the first equality, we can easily describe the whole weight spaces of $\Gamma_{2,0}=\left[\Gamma_{0,2}, \Gamma_{0,2}\right]$. Then the second equality follows from a similar argument. This completes the proof of Theorem 1.3.

\section{The relation with the Casson invariant}

In this section, motivated by Corollary 1.2, we discuss a possible relation between the Jones representation $\rho$ and the Casson invariant of homology 3spheres, and pose a related problem.

We first recall the description of the Casson invariant, denoted by $\lambda$, in terms of mapping class groups of surfaces by Morita. We refer to [13], [14] for further details. As before, let $\Sigma_{g}$ be a closed oriented surface of genus $g \geq 2$, and let $\mathcal{M}_{g}$ be its mapping class group. Also, let $\mathcal{K}_{g}$ be the subgroup of $\mathcal{M}_{g}$ generated by all the Dehn twists along separating simple closed curves on $\Sigma_{g}$. Given a homology 3 -sphere $M$ together with an embedding $f: \Sigma_{g} \rightarrow M$, the Casson invariant gives rise to a homomorphism $\lambda_{f}: \mathcal{K}_{g} \rightarrow \mathbb{Z}$. Roughly, $\lambda_{f}$ is defined by $\lambda_{f}(\psi)=\lambda\left(M_{\psi}\right)-\lambda(M)$ for $\psi \in \mathcal{K}_{g}$ where $M_{\psi}$ is the homology 3 -sphere obtained from $M$ by first cutting along $f\left(\Sigma_{g}\right)$ and then regluing by $\psi$. Furthermore, $\lambda_{f}$ can be decomposed as a sum of two $\mathbb{Q}$-valued homomorphisms:

$$
\lambda_{f}=-\frac{1}{24} d_{1}+q_{f}
$$

Here the homomorphism $d_{1}$ coincides with a secondary invariant which arises from Morita's theory of secondary characteristic classes of surface bundles ([13], [15], [16]). In particular, $d_{1}$ is independent of the choice of the pair $(M, f)$, and is invariant under the conjugation action of $\mathcal{M}_{g}$ on $\mathcal{K}_{g}$. It is known that such a $\mathbb{Q}$-valued homomorphism of $\mathcal{K}_{g}$ is unique up to nonzero scalars ([14, Theorem 5.7]).

On the other hand, the homomorphism $q_{f}$ factors through $\tau_{g}(2)$ and depends on the choice of $(M, f)$.

Now we assume that $g=2$. Note that $\mathcal{K}_{2}=\mathcal{I}_{2}$ as mentioned before. In view of Corollary 1.2, we see that $q_{f}$ factors through $\rho$. Thus we may say that $\rho$ contains the full information of $\lambda$, in the case of $g=2$, if and only if $\rho$ contains the information of $d_{1}$. 
Now we set $\mathcal{Z}=\mathcal{I}_{2} / \operatorname{Ker} d_{1}$, and $\mathcal{Z}_{\sigma}=\sigma\left(\mathcal{I}_{2}\right) / \sigma\left(\operatorname{Ker} d_{1}\right)$ for an arbitrary homomorphism $\sigma$ of $\mathcal{I}_{2}$. Note that $\mathcal{Z}$ is isomorphic to the infinite cyclic group $\mathbb{Z}$ and $\mathcal{Z}_{\sigma}$ is a quotient of $\mathcal{Z}$. Under these notation, the information of $d_{1}$ contained in $\rho$ concentrates on the cyclic group $\mathcal{Z}_{\rho}$. On the other hand, we can easily see that $\operatorname{Ker} d_{1}=\left[\mathcal{M}_{2}, \mathcal{I}_{2}\right]$. We thus have the following:

Proposition 6.1 The homomorphism $d_{1}$ factors through the Jones representation $\rho$ if and only if

$$
\mathcal{Z}_{\rho}=\rho\left(\mathcal{I}_{2}\right) / \rho\left(\left[\mathcal{M}_{2}, \mathcal{I}_{2}\right]\right) \cong \mathbb{Z}
$$

At the moment, we do not know whether the equality (6.1) is true or not.

Problem 6.2 Determine the order of the cyclic group $\mathcal{Z}_{\rho}$.

Remark 6.3 (1) If the order of $\mathcal{Z}_{\rho}$ is finite, then it implies that $\rho$ is not faithful on $\mathcal{I}_{2}$.

(2) Our previous result [10] implies that the order of $\mathcal{Z}_{\rho}$ is at least 10.

(3) For any reduction $r$ of $\rho$, the computation of $\mathcal{Z}_{r}$ would be helpful in estimating the order of $\mathcal{Z}_{\rho}$. With such an expectation, we considered the cases $r=\varphi^{(1)}$ and $r=\varphi^{(2)}$. As a result, we obtained the isomorphisms

$$
\mathcal{Z}_{\varphi^{(1)}}=\mathcal{Z}_{\tau_{2}(2)} \cong \mathbb{Z} / 10, \quad \mathcal{Z}_{\varphi^{(2)}} \cong \mathbb{Z} / 10
$$

by computer calculations.

In view of the above, it remains possible that the order of $\mathcal{Z}_{\rho}$ is exactly 10. In any case, it might be interesting to note that $\mathbb{Z} / 10$ is isomorphic to the abelianization of $\mathcal{M}_{2}$.

\section{References}

[1] M Asada, H Nakamura, On graded quotient modules of mapping class groups of surfaces, Israel J. Math. 90 (1995) 93-113

[2] D Bar-Natan, On the Vassiliev knot invariants, Topology, 34 (1995) 423-472

[3] J S Birman, Mapping class groups of surfaces, AMS Contemporary Mathematics 78 (1988) 13-43

[4] J S Birman, New points of view in knot theory, Bull. Amer. Math. Soc. (N.S.) 28 (1993) 253-287 
[5] J S Birman, H Hilden, Isotopies of homeomorphisms of Riemann surfaces and a theorem about Artin's braid group, Annals of Math. 97 (1973) 424-439

[6] J S Birman, X S Lin, Knot polynomials and Vassiliev's invariants, Invent. Math. 111 (1993) 225-270

[7] W Fulton, J Harris, Representation Theory, Graduate Texts in Math. 129, Springer-Verlag (1991)

[8] S P Humphries, Normal closures of powers of Dehn twists in mapping class groups, Glasgow J. Math. 34 (1992) 313-317

[9] V F R Jones, Hecke algebra representations of braid groups and link polynomials, Annals of Math. 126 (1987) 335-388

[10] Y Kasahara, The Jones representation of genus 2 at the 4 -th root of unity and the Torelli group, preprint

[11] M Lazard, Sur les groupes nilpotents et les anneaux de Lie, Ann. Sci. Ecole Norm. Sup. (3) 71 (1954) 101-190

[12] W Magnus, A Karrass, D Solitar, Combinatorial Group Theory, Interscience Publ. (1966)

[13] S Morita, Casson's invariant for homology 3-spheres and characteristic classes of surface bundles I, Topology, 28 (1989) 305-323

[14] S Morita, On the structure of the Torelli group and the Casson invariant, Topology, 30 (1991) 603-621

[15] S Morita, Casson invariant, signature defect of framed manifolds and the secondary characteristic classes of surface bundles, J. Differential Geometry 47 (1997) 560-599

[16] S Morita, Structure of the mapping class groups of surfaces: a survey and a prospect, from: "Proceedings of the Kirbyfest", Geometry and Topology Monographs, Vol. 2 (1999) 349-406

[17] J Powell, Two theorems on the mapping class group of surfaces, Proc. Amer. Math. Soc. 68 (1978) 347-350

Department of Electronic and Photonic System Engineering, Kochi University of Technology, Tosayamada-cho, Kagami-gun, Kochi, 782-8502 Japan

Email: kasahara@ele.kochi-tech.ac.jp

Received: 18 October 2000 